\title{
Classical and quantum calculations of the temperature dependence of the free energy of argon
}

\author{
Wenwu Xu ${ }^{1,2,3^{*}}$, Andrew P. Horsfield ${ }^{4}$, David Wearing ${ }^{4}$, Peter D. Lee ${ }^{1,2}$ \\ ${ }^{1}$ School of Materials, University of Manchester, Manchester M13 9PL, UK \\ ${ }^{2}$ Research Complex at Harwell, Didcot OX11 OFA, UK \\ ${ }^{3}$ Department of Mechanical Engineering, San Diego State University, San Diego 92182, USA \\ ${ }^{4}$ Department of Materials, Imperial College London, London SW7 2AZ, UK \\ *wenwu.xu@sdsu.edu; Tel: 1-619-594-6068
}

\begin{abstract}
The free energy is central to statistical mechanics and thermodynamics, and its accurate calculation via. computational modeling is important for a large number of applications, especially when its experimental value is hard to obtain. Several established and general methods for calculating the Helmholtz free energy across different length scales, including continuum, atomistic and quantum mechanical, are compared and analyzed. A computational approach is then proposed to calculate the temperature dependences of internal energy and absolute Helmholtz free energy for solid and liquid phases with the coupling of thermodynamic integration (TI) and harmonic approximation calculations from both classical molecular dynamics (MD) and density functional theory (DFT). We use the Lennard-Jones system as an example (i.e. argon) for the demonstration of the approach. It is observed that the free energy transits smoothly from being describable by the harmonic approximation to including anharmonic effects at a transition temperature around $0.56 T_{m}$; below this temperature, the quantum behavior of atoms is important. At higher temperatures $\left(T>0.56 T_{m}\right)$, the TI and harmonic approximation results for the Helmholtz free energy functions become increasingly divergent with the increase of temperature. This work demonstrates that a multiscale approach employing TI, MD, and DFT can provide accurate calculations of the temperature dependence of absolute Helmholtz free energy for both solid and liquid phases.
\end{abstract}


Keywords: Thermodynamic integration; Harmonic approximation; Molecular dynamics;

Quantum mechanics; Helmholtz free energy; Argon.

\section{Introduction}

The Helmholtz (volume constant) or Gibbs (pressure constant) free energy of a system is one of the most important thermodynamic quantities for condensed-phase systems [1]; it determines what the stable structures are, and the direction changes take. Bulk free energies can be derived from experiments, but interfacial free energies are much harder to obtain. While the calculation of bulk free energies and derivative thermodynamic quantities, and static energies for interfaces, are common and widespread applications of atomistic simulation techniques [2-4], the computation of solid-liquid interfacial free energies is still challenging.

Free energy functions can be used for studying important fundamental problems in materials sciences, such as phase and structural transitions [5,6], interfacial energies $[7,8]$, or other critical phenomena [9-11]. There are several techniques available for calculating the absolute or relative free energy as a function of temperature of a system, an attractive option when experimental determination is difficult. In particular, we may obtain the crystal-melt (or solidliquid) interfacial free energy as a function of temperature for systems in which the liquid and solid are formed from materials with different melting points based on the free energy functions of the solid and liquid phases. The interfacial energy would be the difference between the total free energy for a system with a solid-liquid interface, and the free energies of the bulk solid and liquid components [8]. In this case, the accurate computations of free energies for solid and liquid bulk phases become critical. Note that for the solid-liquid interface in a single material at its melting point, special methods are needed $[12,13]$. 
At a temperature sufficiently far below the melting point, one can compute absolute free energies quite accurately using the harmonic approximation (HA) [1]. To get free energies at higher temperatures, in particular when one of the materials is in the liquid state, one might perform thermodynamic integration (TI) from a lower temperature to a higher one. A problem with the usual TI approach is that one cannot integrate through the phase transition to reach the liquid state, thereby obtaining an absolute free energy for the liquid. The difficulty is that at the melting temperature there is a discontinuity in the internal energy on going from the solid to the liquid states (the latent heat of melting) which makes it impossible to carry out a reversible calculation: there is a temperature that we must include at which the internal energy is not uniquely defined.

One way around this is to note that the free energies of the solid and liquid states are identical at the melting point. We can find the absolute free energy of the solid using available techniques to temperatures slightly above the melting point (superheated solid), and find the relative free energies of the liquid to just below the melting point (supercooled liquid). We can then match the free energies at the melting point. To do this we need techniques that allow us to compute the various contributions, all of which exist.

In this work, as a first step towards computing solid-liquid interfacial energies, we investigate some of these techniques of computing free energies of solid and/or liquid bulk phases and how these different methods can be combined. In particular we combine TI for classical molecular dynamics (MD) simulations with lattice dynamics calculations of the phonon spectrum. This allows us to compute the temperature dependence of the Helmholtz free energy of both the solid and liquid phases by using a Lennard-Jones system (argon) as an example. In Section 2 we introduce the method and modeling procedure which involves 3 aspects: (i) determination 
of the melting point; (ii) temperature dependence of the internal energy functions for both solid and liquid phases; (iii) a reference value of an absolute free energy at a specified temperature far below the melting point. In Section 3 we combine this information to produce the Helmholtz free energy as a function of temperature over a large temperature range, spanning both the solid and liquid states. We end with a summary of conclusions in Section 4.

\section{Method and Simulations}

Suppose we know the internal energy of a system $U$ as a function of temperature $T$, and wish to compute the free energy $F_{2}$ at temperature $T_{2}$ given that we know the free energy $F_{1}$ at temperature $T_{1}$. The Gibbs-Helmholtz equation then gives

$$
\frac{F_{2}}{T_{2}}-\frac{F_{1}}{T_{1}}=-\int_{T_{1}}^{T_{2}} \frac{U}{T^{2}} d T
$$

Thus, to calculate the absolute Helmholtz free energy as a function of temperature $F(T)$, we need to know its value at a reference temperature $T_{R}$, which we shall obtain using the result for a collection of quantum harmonic oscillators. Eq. (1) can now be written as

$$
\frac{F(T)}{T}=\frac{F_{R}}{T_{R}}-\int_{T_{R}}^{T} \frac{U\left(T^{\prime}\right)}{T^{\prime 2}} d T^{\prime}
$$

As noted above, we emphasise that the temperature function of internal energy is not continuous across the solid/liquid transition (i.e. at the melting point, $T_{m}$ ), whereas $F(T)$ is continuous because the free energies of solid and liquid are equal at the melting temperature. The superscript prime symbol on temperature $\left(T^{\prime}\right)$ corresponds to classical molecular dynamics simulations, and it is different from the temperature $(T)$ in quantum calculations. This is because, at temperatures close to $0 \mathrm{~K}$, there is a discrepancy between the temperatures used for classical molecular dynamics and for lattice dynamics (quantum) calculations, which originates from the quantum zero point energy [14]. This discrepancy, however, becomes small when the temperature of the crystal system exceeds $\sim 0.1 T_{\mathrm{m}}$ ( $T_{\mathrm{m}}$ is the melting point) [14]. In summary, using Eq. (2), one can easily calculate the temperature dependence of the Helmholtz free energy 
of a system provided a value can be found for a reference temperature, and the internal energy as a function of temperature can be found for both the solid $\left(T_{R}<T \leqslant T_{m}\right)$ and liquid $\left(T \geqslant T_{m}\right)$ states.

There is one important technical point that we investigate here. At low temperatures, the atomic motion is quantum in nature, and thus we use a quantum mechanical expression suitable for harmonic motion. To extrapolate above low temperatures, we have to incorporate anharmonic terms, and the most straightforward way to do that is to perform TI with MD. However, for MD the atoms are treated as classical objects. Thus, to reach higher temperatures we simultaneously move from harmonic to anharmonic motion, and from a quantum to a classical description of the atoms. For this to work, the atoms need to be describable by classical mechanics before the harmonic approximation fails. Our calculations suggest the transition occurs smoothly.

A first step for this method is the accurate determination of the melting point. Here we obtain an accurate value of the melting point of argon by allowing the solid and liquid phases to reach equilibrium in a constant energy MD simulation [15]. Then, we compute the internal energies at a series of temperatures for both solid $\left(10 \mathrm{~K}<T \leqslant T_{m}\right)$ and liquid $\left(T \geqslant T_{m}\right)$ argon using constant volume and energy (NVE) MD simulations; constant temperature and pressure (NPT) MD simulations are used to establish a suitable volume. Lastly, the absolute value of the Helmholtz free energy at $T_{R}=10 \mathrm{~K}$ (the chosen reference temperature in the current work) is calculated using the standard expression that relates the free energy to the phonon frequency spectrum [16],

$$
F(T)=E_{t o t}(T)+k_{B} T \int F(\omega) \ln \left[1-\exp \left(-\frac{\hbar \omega}{k_{B} T}\right)\right] d \omega
$$


where the frequencies $\omega$ are computed from the dynamical matrix in quantum calculations. Here $E_{\text {tot }}(T)$ includes the contribution from zero-point energy. $k_{B}$ and $\hbar$ are the Boltzmann and Planck constant, respectively. $F(\omega)$ is the phonon density of states. Based on the above calculations using Eqs. (1)-(3) one can compute the temperature dependence of the Helmholtz free energy for argon with the temperature ranging from the low reference temperature $\left(e . g . T_{R}\right.$ $=10 \mathrm{~K})$ to temperatures well above the melting point $\left(T \geqslant T_{m}\right)$.

\subsection{MD simulations: melting point of argon}

If not specified otherwise, our classical MD simulations are carried out by using an open-source code DL_POLY, which has been developed at Daresbury Laboratory by I.T. Todorov and W. Smith [17]. The Lennard-Jones $12-6$ potential (with $\varepsilon=0.0104 \mathrm{eV}$ and $\sigma=3.4 \AA$ [18]) is employed to define the interactions between pairs of argon atoms. We determine the melting point of argon at atmospheric pressure $(P=1.0 \mathrm{~atm}$. $)$ by constructing a pressure versus temperature diagram from a series of solid/liquid co-existence simulations using the NVE (constant volume and energy) ensemble. These solid/liquid co-existence interface models equilibrate at a certain temperature and pressure, which is, by definition, the melting point at that pressure [15].

To perform one of these solid/liquid equilibrium simulations, we first create an initial configuration for the solid/liquid interface model that consists of about $50 \%$ solid by volume (42,592 atoms) with the rest being liquid (32,000 atoms): see below for a description of how the liquid is generated. These systems are pre-equilibrated at $10 \mathrm{~K}$ and $85 \mathrm{~K}$ (slightly higher than the experimental melting point of argon: $83.8 \mathrm{~K}$ [19]), respectively. Fig. 1(a) shows the front-view (projection of $x-z$ plane) of a 3-D interface model, with the solid/liquid boundary indicated by a long-dashed line, perpendicular to the $z$-axis. This initial interface model is then relaxed and equilibrated at three different temperatures of $81 \mathrm{~K}, 83 \mathrm{~K}$, and $85 \mathrm{~K}$ within the 
NVT (constant volume and temperature) ensemble for $10 \mathrm{~ns}$ (i.e. 10 million steps with $1 \mathrm{fs}$ as the time step of the MD simulations). Fig. 1(b) shows an equilibrated configuration of the interface system at $81 \mathrm{~K}$. The solid/liquid boundary remains, but there is shrinkage of the liquid volume when the temperature is decreased from $85 \mathrm{~K}$ to $81 \mathrm{~K}$. In addition, the solid/liquid boundary also remains when the interface system is equilibrated at $83 \mathrm{~K}$ and $85 \mathrm{~K}$ (figures are not shown here) with larger volume fraction of liquids as compared with that for the case of 81 $\mathrm{K}$. We now have three pairs of temperature-pressure values through averaging the values of temperature and pressure using the last $5 \mathrm{~ns}$ of the simulation results (out of $10 \mathrm{~ns}$ ). In Fig. 2, the closed shapes of round, rhombus, and triangle represent the three pairs of temperaturepressure values corresponding to $81 \mathrm{~K}, 83 \mathrm{~K}$ and $85 \mathrm{~K}$, respectively.

To allow for accurate interpolation to a specific pressure, we need to increase the number of points on our pressure versus temperature diagram. To add those points, a number of volumetric perturbations are applied to each interface system equilibrated at the three different temperatures. This is achieved for each case by adding an increment to the cell parameter along the $z$ axis of $0.5 \AA, 1.0 \AA, 1.5 \AA, 2.0 \AA$ and $2.5 \AA$. As a result, a thin slab of vacuum with certain thickness perpendicular to the $z$ axis is added for each volumetric perturbation. We then perform NVE simulation for 20 ns to allow the perturbed systems to relax sufficiently. Note that the volumetric perturbations are specifically chosen such that the solid/liquid boundary remains after relaxation. By averaging the values of temperature and pressure from each NVE simulation from the last $5 \mathrm{~ns}$ (out of $20 \mathrm{~ns}$ ), we are able to construct the pressure versus temperature diagram of solid/liquid co-existence systems. The results are represented by closed (un-perturbed) and open (perturbed) shapes shown in Fig. 2. The pressure-temperature diagram can now be used to determine the melting point of argon corresponding to a particular pressure. Specifically, the melting point of argon at 1.0 atm. is found to be $81.0 \pm 0.1 \mathrm{~K}$, i.e. the crossing 
point (as indicated by the solid arrow) of the dashed line (1.0 atm.) and dotted line (the linear fit to all pairs of pressure-temperature values), which is in good agreement with experiments (83.8 K [19]) and previously reported modelling results (82.8 K [15], 81.5 K [20]).

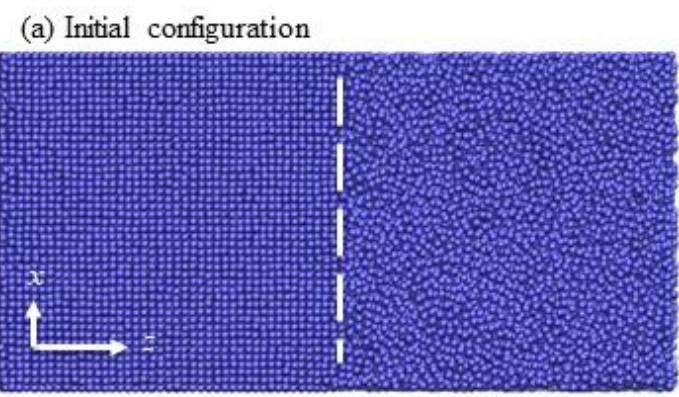

(b) Equilibrium configuration

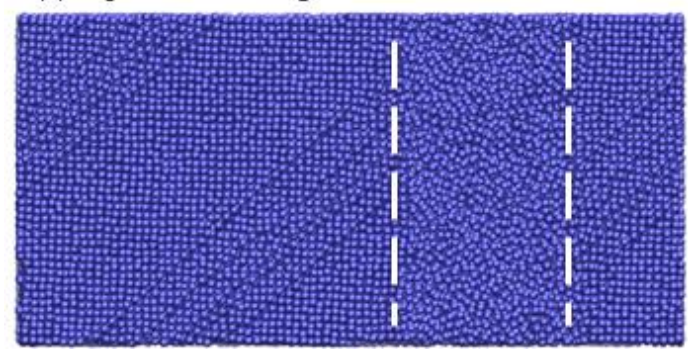

Fig. 1. (Color Online) MD models of solid/liquid interface system of argon: (a) the initial configuration consisting of equal volumes of solid and liquid bulk phases; (b) an equilibrium configuration of the initial solid/liquid interface system following NVT relaxation at $81 \mathrm{~K}$ with a simulation time of $10 \mathrm{~ns}$. Note that the white long-dashed lines in (a) and (b) indicate the boundaries between solid and liquid phases.

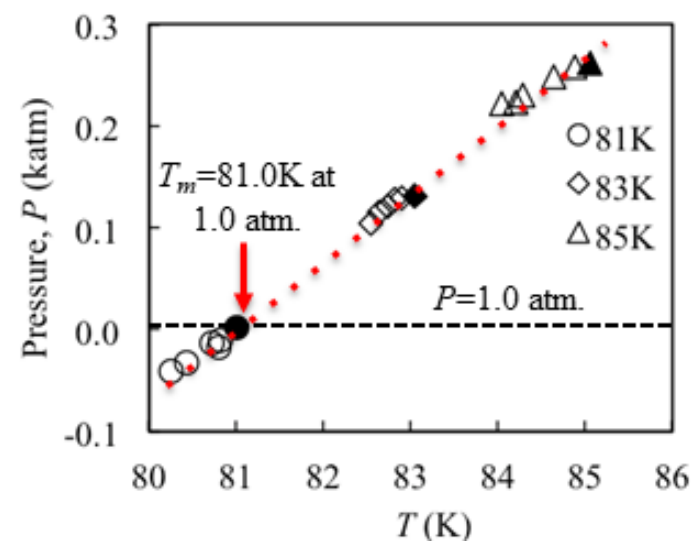

Fig. 2. (Color Online) Pressure-temperature diagram for the solid/liquid co-existing interface systems, created using molecular dynamics simulations. The melting point $\left(T_{\mathrm{m}}\right)$ of argon at a 
pressure of $1.0 \mathrm{~atm}$. is determined from the crossing point of the black dashed line $(P=1.0$ atm.) and the red dotted line (linear fit to the values of pressure vs. temperature).

\subsection{MD simulations: internal energies of solid and liquid argon}

We now compute the internal energies as a function of temperature for both solid $(10 \mathrm{~K}<T \leqslant$ $\left.T_{m}\right)$ and liquid $\left(T \geqslant T_{m}\right)$ argon using MD simulations. To do that, we create a supercell of crystal argon containing 32,000 atoms. The system is then subjected to an MD simulation using the NPT ensemble for $200 \mathrm{ps,}$, which brings the system pressure to $1.0 \mathrm{~atm}$. at a temperature of $10 \mathrm{~K}$. Subsequently, the equilibrated crystal argon is relaxed within an NVE ensemble at a series of temperatures from $10 \mathrm{~K}$ up to the determined melting point of argon (i.e. $81 \mathrm{~K}$ at 1 atm.). To create bulk liquid argon we use two steps: (i) randomization and (ii) equilibration. Randomization is achieved by heating up the supercell of crystal argon (containing 32,000 atoms) in the simulation box with an extra $10 \%$ volume of vacuum inserted. Equilibration is then achieved when the randomized system is brought to a pressure of $1 \mathrm{~atm}$. and a temperature of $85 \mathrm{~K}$ by performing MD in the NPT ensemble. Using the pre-equilibrated liquid argon at 85 $\mathrm{K}$ and $1 \mathrm{~atm}$. as a starting point, we then carry out NVE simulations at a series of temperatures from the melting point up to $85 \mathrm{~K}$. A simulation time of $50 \mathrm{ps}$ is found to be sufficient to equilibrate the crystal and liquid argon models at each temperature.

We compute the internal energies of solid and liquid argon at each temperature by averaging the values of internal energy over the last $10 \mathrm{ps}$ out of a total $50 \mathrm{ps}$ of the NVE simulations. The values of the internal energy obtained from the MD simulations of solid and liquid argon are shown in Fig. 3a (red filled-square symbols). The regional enlargements of the red dashed rectangles in Fig. 3a are shown in Fig. 3b and Fig. 3c, respectively. These MD simulated internal energies evaluated for a range of temperatures of the solid and liquid bulk phases will be used to compute the temperature dependence of the absolute Helmholtz free energy of argon 
using thermodynamic integration (see Eq. (2)), as described in Section 3. Internal energies of both solid and liquid argon vary approximately linearly with temperature. Note that there is a discontinuity in the internal energy from the solid state to the liquid state of argon at the melting point.

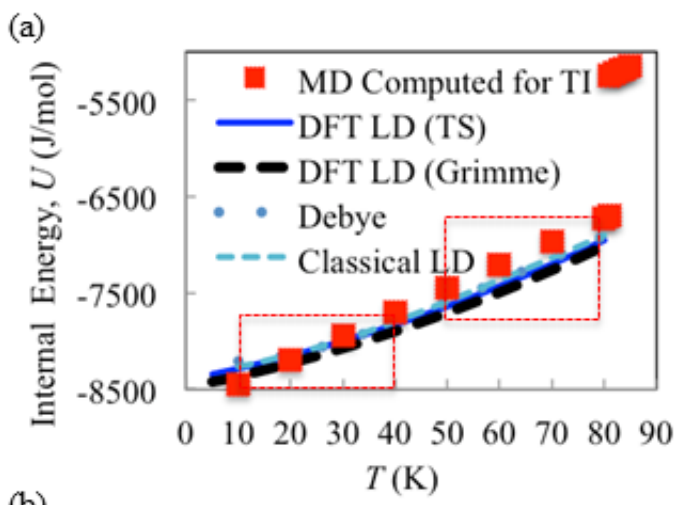

(b)

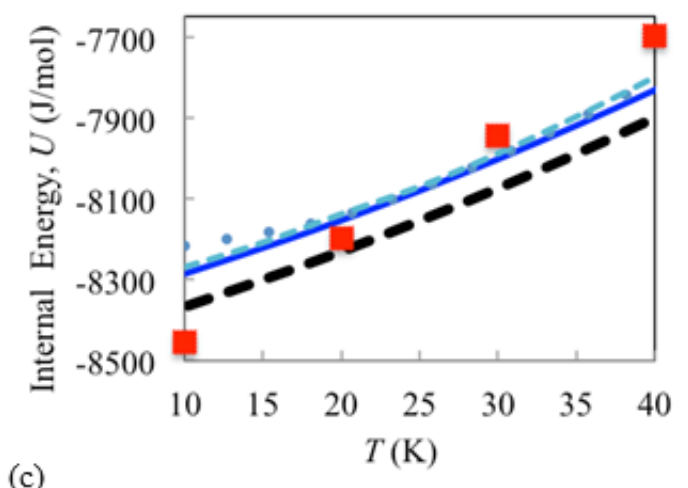

(c)

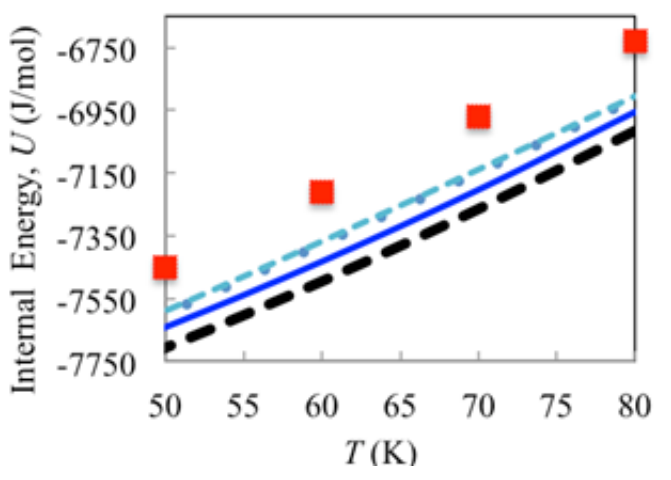

Fig. 3. (Color Online) (a) Internal energy ( $U$, unit: $\mathrm{J} / \mathrm{mol}$ ) as a function of temperature ( $T$, unit: K) of solid argon computed by: molecular dynamics simulations to be used for thermodynamic integration (MD Computed for TI, red filled-square symbols); density functional theory lattice dynamics (DFT LD) with TS (blue solid curve) and Grimme (black long-dashed curve) methods accounting for the van del Walls corrections; classical LD based on molecular 
dynamics (light-blue dashed curve); Debye model (dark-blue dotted curve). (b) and (c) are the local enlargements of (a) at certain low and high temperature regions, respectively, see the red dashed boxes in (a).

\subsection{Harmonic approximation calculations for solid argon}

It is possible to compute the internal energy and Helmholtz free energy as a function of temperature in a harmonic approximation (HA) from the phonon dispersion (or vibrational spectrum) of a solid [1]. Using lattice dynamics (LD) simulations [16], the phonon dispersion of a solid can be computed from the matrix of second derivatives of the energy with respect to atomic displacement. In the following, we use both the Lennard-Jones potential (Classical LD) and density functional theory (DFT LD) to compute the phonon dispersion of solid argon.

In the case of classical LD, the phonon dispersion of solid argon is computed by evaluating the eigenvalues of the dynamical matrix that is constructed by observing the displacements of atoms in a periodic lattice during MD simulations, making use of the fluctuation-dissipation theorem [21]. It should be noticed that we carried out classical LD simulations by using the Large-scale Atomic/Molecular Massively Parallel Simulator (LAMMPS) [22] open-source code that is capable of yielding the phonon dispersion accurately [21]. Phonon calculations by classical LD for solid argon are performed over the entire first Brillouin zone with the modelled phonon spectrum including several high symmetry points $(\Gamma, \mathrm{X}, \mathrm{K}, \mathrm{L})$, as presented in Fig. 4 (light-blue dashed curve). The result of classical LD agrees well with the experimental measurements (filled-circles [23,24], squares [25,26]).

For DFT calculations, we start from the relaxed crystal structure, and compute the phonon dispersion of the solid argon by employing the linear response method (or density functional perturbation theory [16]) with dispersion corrections [27,28], including the TS [27] and 
Grimme [28] approaches. The DFT LD phonon dispersions with TS (blue solid curve) and Grimme (black long-dashed curve) methods are shown in Fig. 4. The DFT results are in good agreement with that from the classical LD (light-blue dashed curve) and experimental measurements: filled-circles [23,24] and squares [25,26] in Fig. 4.

Note that in the above DFT simulations we treat the argon crystal using a primitive face-centred cubic (FCC) cell containing one argon atom. All DFT calculations are performed using the plane wave code Cambridge Serial Total Energy Package (CASTEP) [29]. The Perdew-BurkeErnzerhof (PBE) functional [30] is used for the exchange and correlation energy. Brillouin zone integration is performed using k-points on a Monkhorst-Pack grid [31]. The BroydenFletcher-Goldfarb-Shannon (BFGS) algorithm [32] is applied to relax the atomic position and cell vectors automatically to achieve the minimum total energy of the system. A normconserving pseudo-potential is used with a plane-wave basis set energy cut-off of $390 \mathrm{eV}$. A k-point mesh with spacing of $0.05 \AA^{-1}$ in the reciprocal space is used for all the calculations. Self-consistent field (SCF) calculations are converged to $5 \times 10^{-7} \mathrm{eV} /$ atom for the total energy calculations. The geometry optimization is run until the atomic forces are below $0.01 \mathrm{eV} / \AA$. The optimized lattice parameter of argon crystal is $5.38 \pm 0.01 \AA$ at the ground state, which is in good agreement with that from the experimental extrapolation of $5.31 \AA$ at $0 \mathrm{~K}$ [33].

Statistical mechanics calculations within the HA provide a robust way of calculating the temperature dependences of internal energy and Helmholtz free energy of a solid [16], especially at temperatures far below the melting point $[34,35]$. We thus use this method to compute the internal energy and the Helmholtz free energy as a function of temperature for the solid argon, from which the value of free energy $\left(F_{R}\right)$ at a reference temperature $\left(e . g\right.$. at $T_{R}=$ 
$10 \mathrm{~K})$ can be obtained. It should be noted that the van der Waals interactions plays an important role in the solid argon [36], and so needs to be included in the DFT calculations.

For comparison purpose, we also compute the internal energy as a function of temperature using the analytical Debye model [1]. The Debye characteristic temperature used in the model calculations is $85 \mathrm{~K}$ from experimental measurement [37]. The results of the harmonic approximation of classical and DFT LD, as well as the analytic Debye model, for the internal energy of solid argon are shown in Fig. 3. As noted before, the discrepancy between the temperatures used for classical molecular dynamics and for lattice dynamics is small when the temperature of the crystal system exceeds $\sim 0.1 T_{\mathrm{m}}$ ( $T_{\mathrm{m}}$ is the melting point) [14]. Thus, for the Ar crystal phase, we compare our results from classical MD and lattice dynamics for temperatures above $10 \mathrm{~K}$, which is greater than $0.1 T_{\mathrm{m}}$ as the melting point of $\mathrm{Ar}$ is $81 \mathrm{~K}$.

The four results (i.e. DFT LD (TS), DFT LD (Grimme), Debye, and Classical LD) for the internal energy as a function of temperature of solid argon have very similar gradients over a large temperature range, with a small energy shift between them $(<0.1 \mathrm{~kJ} / \mathrm{mol})$. Interestingly, at relatively low temperatures (e.g. $T<40 \mathrm{~K}$, see Fig. 3(b)) the gradients of the four internal energy functions based on quantum atoms and the HA, are distinctly different from that of the classical MD simulations (red filled-squares in Fig. 3, details of the simulations described in Section 2.2). Note that the MD computed internal energy will be used to calculate the Helmholtz free energy by thermodynamic integration calculations (see Section 3). The difference of gradient of the internal energy function (see Fig. 3) is because of the quantum behavior of the atoms matters at low temperatures (e.g. $T<40 \mathrm{~K}$ for the case of solid argon). At higher temperatures (e.g. $T>50 \mathrm{~K}$, see Fig. 3(c)) all five results for the internal energy function have almost the same slope. This is as expected: they should have the same heat 
capacity close or equal to $3 \mathrm{R}$ (where $\mathrm{R}$ is the ideal gas constant) above a certain temperature. This justifies a switch from the quantum harmonic model to TI somewhere between $40 \mathrm{~K}$ and $50 \mathrm{~K}$.

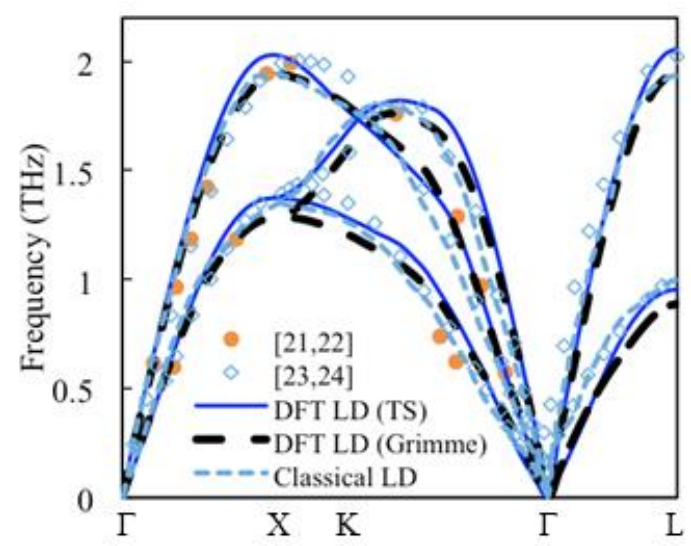

Fig. 4. (Color Online) Phonon dispersion of solid argon by various approaches: density functional theory (DFT LD) with TS (blue solid curve) and Grimme (black long-dashed curve) methods accounting for the van der Waal dispersion corrections, and classical LD (light-blue dashed curve). Filled-circles and squares represent experimental measurements reported in the literature.

\section{Temperature dependence of Helmholtz free energy of solid and liquid argon}

The harmonic approximation calculations also provide an accurate description of the temperature dependence of the Helmholtz free energy for solid argon at temperatures far below the melting point [1]. Fig. 5(a) shows the Helmholtz free energy as a function of temperature of solid argon that is computed from the harmonic approximation calculations based on different approaches. The results of density functional theory lattice dynamics (DFT LD) with TS and Grimme methods are shown by the blue solid curve and black long-dashed curve in Fig. 5(a), respectively. The Helmholtz free energy function according to the analytical Debye model and the L-J potential based classical lattice dynamics (Classical LD) are indicated by the dark-blue dotted curve and light-blue dashed curve, respectively. Within the harmonic 
approximation, the DFT results of Helmholtz free energy function (DFT LD with TS and Grimme methods) are in good agreement with the Debye and Classical LD results at certain low temperature range (e.g. $T<40 \sim 50 \mathrm{~K})$. At higher temperatures, the discrepancy becomes significant and increases with the increase of the temperature.

We then perform thermodynamic integration to obtain the Helmholtz free energy as a function of temperature for both solid and liquid argon based on Eq. (1). We use the DFT harmonic approximation calculations (i.e. DFT LD (TS)) to provide a reference value of Helmholtz free energy $\left(F_{R}\right)$ at a certain temperature $\left(\right.$ e.g. $\left.T_{R}=10 \mathrm{~K}\right)$. Thermodynamic integration (see Eq. (1)) based on MD simulations is then used to advance to higher temperatures. Now, we have: (i) the melting point $\left(T_{m}=81 \mathrm{~K}\right)$ of argon (Fig. 2); (ii) the internal energy functions $(U(T))$ of solid and liquid argon from MD modelling (Fig. 3); (iii) a value of Helmholtz free energy at a reference temperature (i.e. $F_{R}$ at $T_{R}=10 \mathrm{~K}$ ) from DFT harmonic approximation calculations (Fig. 5). We can now perform the thermodynamic integration in order to compute the temperature dependence of the Helmholtz free energy for both solid and liquid argon based on our MD simulations by using Eq. (1).

For example, the Helmholtz free energy as a function of temperature over the range of $10 \mathrm{~K}$ to $85 \mathrm{~K}$ is computed by taking the reference value at $10 \mathrm{~K}$ from the DFT LD (TS) calculation; the result is shown by the red solid curve in Fig. 5(a). The Helmholtz free energy at the melting point (as indicated by the vertical black dashed arrow in Fig. 5(a)) is equal for solid and liquid argon. A continuous transition in the Helmholtz free energy from solid to liquid can thus be obtained. The Helmholtz free energies of argon in this work are in reasonable agreement with reported values obtained by Monte Carlo methods (filled yellow circles [38] and blue triangles [39]) and the data based on the experimentally measured equation of state (EOS) of argon 
(National Institute of Standards and Technology, NIST), as indicated by the red diamond $[40,41]$.

It is interesting to note that the Helmholtz free energy function from classical TI agrees well with that from both the classical and DFT harmonic calculations at low temperature, but diverges when the temperature is increased to higher values. Fig. 5(b) shows the differences in Helmholtz free energy as a function of temperature between classical TI $\left(F_{\mathrm{CTI}}\right)$ and harmonic calculations by DFT $\left(F_{\mathrm{DFT}(\mathrm{TS})}\right)$ and classical $\left(F_{\mathrm{CLD}}\right)$ lattice dynamics, as shown by the red solid curve $\left(F_{\mathrm{CTI}}-F_{\mathrm{DFT}(\mathrm{TS})}\right)$ and black dashed curve $\left(F_{\mathrm{CTI}}-F_{\mathrm{CLD}}\right)$ in Fig. 5(b), respectively. The difference begins to become obvious above about $45 \mathrm{~K}$ (i.e. $0.56 T_{\mathrm{m}}$, as indicated by the vertical black dashed arrow in Fig. 5(b)), and then becomes increasingly larger with a further increase of temperature. This suggests that the free energy calculated using the harmonic approximation is only reliable when the temperature is sufficiently below the melting point of a solid crystal system (e.g. $0.56 T_{\mathrm{m}}$ in the case of solid argon). 


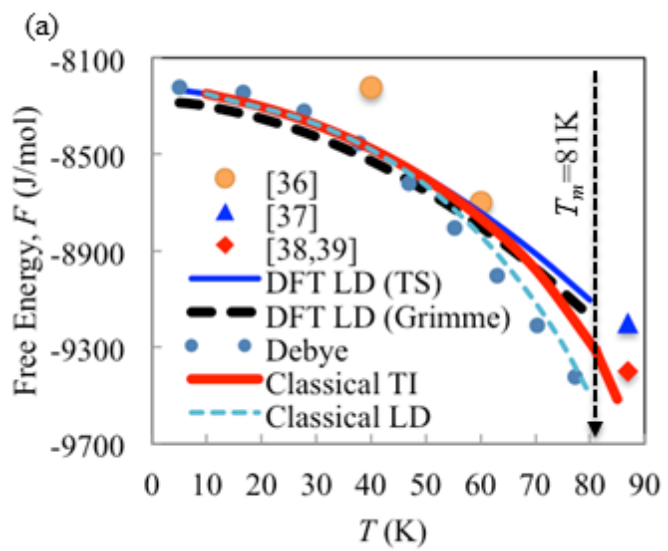

(b)

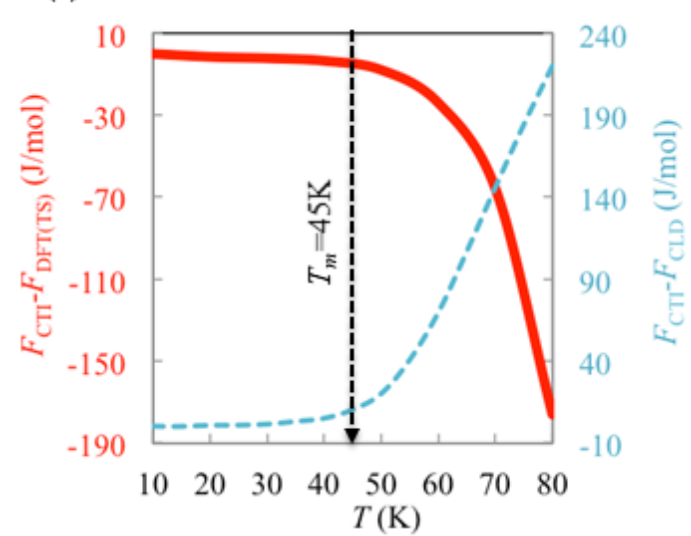

Fig. 5. (Colour Online) (a) Helmholtz free energy $(F)$ as a function of temperature $(T)$ of argon computed by different approaches: density functional theory lattice dynamics (DFT LD) with TS (blue solid curve) and Grimme (black long-dashed curve) methods accounting for the van der Waal dispersion corrections; classical LD (light-blue dashed curve); thermodynamic integration based on classical molecular dynamics simulations (Classical TI, red solid curve); Debye model (dark-blue dotted curve). (b) Free energy deviations as a function of temperature with respect to Helmholtz free energy by Classical TI: DFT LD (TS) $\left(F_{\mathrm{CTI}}-F_{\mathrm{DFT}(\mathrm{TS})}\right.$, red solid curve) and classical LD ( $F_{\mathrm{CTI}}-F_{\mathrm{CLD}}$, black dashed curve).

\section{Conclusion}

By using a combination of statistical mechanics in the harmonic approximation (using a phonon spectrum computed by DFT), and thermodynamic integration (using classical potentials), we have computed the temperature dependence of the Helmholtz free energy for argon (from 10 
$\mathrm{K}$ to $85 \mathrm{~K})$. The results from thermodynamic integration and the harmonic approximation for the Helmholtz free energy of solid argon are rather similar for $T<0.56 T_{m}$ but become increasingly divergent with further increase of temperature. We thus confirmed that free energy calculations using the harmonic approximation are only accurate when the temperature is sufficiently below the melting point of a solid system (e.g. $0.56 T_{\mathrm{m}}$ in the case of solid argon), above which anharmonic contributions to the free energy become increasingly important. The accurate determination of free energy as a function of temperature for both solid and liquid systems can provide an important fundamental basis for investigating various thermodynamic phenomena, but especially solid-liquid interfacial free energies.

\section{Acknowledgements}

The authors wish to acknowledge financial support from the ExoMet Project (which is cofunded by the European Commission in the 7th Framework Programme, contract FP7-NMP3LA-2012-280421, the European Space Agency, and by the individual partner organizations), the EPSRC (EP/I02249X/1) and the Research Complex at Harwell. This work made use of the facilities of N8 HPC provided and funded by the N8 consortium and EPSRC (Grant No.EP/K000225/1), and the Imperial College London High Performance Computing service. APH also gratefully acknowledges support from the Thomas Young Centre under grant TYC10.

\section{References}

[1] C. Kittel, Introduction to Solid State Physics, 2004.

[2] R. Freitas, M. Asta, M. De Koning, Nonequilibrium free-energy calculation of solids using LAMMPS, Comput. Mater. Sci. 112 (2016) 333-341.

[3] M. Athènes, M.C. Marinica, Free energy reconstruction from steered dynamics without post-processing, J. Comput. Phys. 229 (2010) 7129-7146.

[4] L. Cao, G. Stoltz, T. Lelièvre, M.C. Marinica, M. Athènes, Free energy calculations from adaptive molecular dynamics simulations with adiabatic reweighting, J. Chem. 
Phys. 140 (2014) 129903.

[5] W. Xu, X. Song, N. Lu, C. Huang, Thermodynamic and experimental study on phase stability in nanocrystalline alloys, Acta Mater. 58 (2010) 396-407.

[6] Y. Peng, F. Wang, Z. Wang, A.M. Alsayed, Z. Zhang, A.G. Yodh, Y. Han, Two-step nucleation mechanism in solid-solid phase transitions., Nat. Mater. 14 (2015) 101-108.

[7] J. Wang, A. Horsfield, P.D. Lee, P. Brommer, Heterogeneous nucleation of solid Al from the melt by Al3 Ti: Molecular dynamics simulations, Phys. Rev. B - Condens. Matter Mater. Phys. 82 (2010) 144203.

[8] D. Wearing, A.P. Horsfield, W. Xu, P.D. Lee, Which wets TiB2 inoculant particles: Al or Al3Ti?, J. Alloys Compd. 664 (2016) 460-468.

[9] Y. Yang, M. Asta, B.B. Laird, Solid-Liquid Interfacial Premelting, Phys. Rev. Lett. $110(2013) 96102$.

[10] W. Xu, X. Song, Z. Zhang, Thermodynamic study on metastable phase: From polycrystalline to nanocrystalline system, Appl. Phys. Lett. 97 (2010) 181911.

[11] W. Xu, X. Song, N. Lu, M. Seyring, M. Rettenmayr, Nanoscale thermodynamic study on phase transformation in the nanocrystalline Sm2Co17 alloy., Nanoscale. 1 (2009) $238-244$.

[12] S. Angioletti-Uberti, M. Asta, M.W. Finnis, P.D. Lee, Solid-liquid phase equilibria from free-energy perturbation calculations, Phys. Rev. B. 78 (2008) 134203.

[13] S. Angioletti-Uberti, M. Ceriotti, P.D. Lee, M.W. Finnis, Solid-liquid interface free energy through metadynamics simulations, Phys. Rev. B. 81 (2010) 125416.

[14] C.Z. Wang, C.T. Chan, K.M. Ho, Tight-binding molecular-dynamics study of phonon anharmonic effects in silicon and diamond, Phys. Rev. B. 42 (1990) 11276-11283.

[15] Y. Zhang, E.J. Maginn, A comparison of methods for melting point calculation using molecular dynamics simulations, J. Chem. Phys. 136 (2012) 144116.

[16] S. Baroni, S. De Gironcoli, A. Dal Corso, P. Giannozzi, Phonons and related crystal properties from density-functional perturbation theory, Rev. Mod. Phys. 73 (2001) $515-562$.

[17] I.T. Todorov, W. Smith, K. Trachenko, M.T. Dove, DL_POLY_3: new dimensions in molecular dynamics simulations via massive parallelism, J. Mater. Chem. 16 (2006) 1911.

[18] S. Lovesey, T. Springer, Dynamics of Solid and Liquids by Neutron Scattering, Springer-Verlag, Berlin, 1977.

[19] D.R. Lide, CRC Handbook of Chemistry and Physics, 2013.

[20] A. Bizjak, T. Urbic, V. Vlachy, Phase diagram of the lennard-jones system of particles from the cell model and thermodynamic perturbation theory, Acta Chim. Slov. 56 (2009) 166-171.

[21] L.T. Kong, Phonon dispersion measured directly from molecular dynamics 
simulations, Comput. Phys. Commun. 182 (2011) 2201-2207.

[22] S. Plimpton, Fast Parallel Algorithms for Short-Range Molecular Dynamics, J. Comput. Phys. 117 (1995) 1-19.

[23] N.P. Gupta, Interpretation of phonon dispersion in solid argon and neon, J. Solid State Chem. 5 (1972) 477-480.

[24] H. Egger, M. Gsanger, Phonon dispersion measurements on an argon single crystal at 4.2 K, Phys. Lett. A. 28 (1968) 433-434.

[25] B. Van Troeye, M. Torrent, X. Gonze, Interatomic force constants including the DFTD dispersion contribution, Phys. Rev. B. 93 (2016) 144304.

[26] Y. Fujii, N. Lurie, R. Pynn, G. Shirane, Inelastic neutron scattering from solid 36Ar, Phys. Rev. B. 10 (1974) 3647.

[27] S. Appalakondaiah, G. Vaitheeswaran, S. Lebègue, N.E. Christensen, A. Svane, Effect of van der Waals interactions on the structural and elastic properties of black phosphorus, Phys. Rev. B. 86 (2012) 35105.

[28] S. Grimme, Density functional theory with London dispersion corrections, Wiley Interdiscip. Rev. Comput. Mol. Sci. 1 (2011) 211-228.

[29] S.J. Clark, M.D. Segall, C.J. Pickard, P.J. Hasnip, M.I.J. Probert, K. Refson, M.C. Payne, First principles methods using CASTEP, Zeitschrift Für Krist. 220 (2005) 567570.

[30] J. Perdew, K. Burke, M. Ernzerhof, Generalized Gradient Approximation Made Simple., Phys. Rev. Lett. 77 (1996) 3865-3868.

[31] H. Monkhorst, J. Pack, Special points for Brillouin zone integrations, Phys. Rev. B. 13 (1976) 5188-5192.

[32] B.G. Pfrommer, M. Côté, S.G. Louie, M.L. Cohen, Relaxation of Crystals with the Quasi-Newton Method, J. Comput. Phys. 131 (1997) 233-240.

[33] O.G. Peterson, D.N. Batchelder, R.O. Simmons, Measurements of X-ray lattice constant, thermal expansivity, and isothermal compressibility of argon crystals, Phys. Rev. 150 (1966) 703-711.

[34] W. Xu, A.P. Horsfield, D. Wearing, P.D. Lee, Diversification of MgO//Mg interfacial crystal orientations during oxidation: A density functional theory study, J. Alloys Compd. 688 (2016) 1233-1240.

[35] W. Xu, A.P. Horsfield, D. Wearing, P.D. Lee, First-principles calculation of $\mathrm{Mg} / \mathrm{MgO}$ interfacial free energies, J. Alloys Compd. 650 (2015) 228-238.

[36] M.B. Doran, The multipole long-range (Van der Waals) interaction coefficients for neon, argon, krypton and xenon, J. Phys. B At. Mol. Phys. 7 (1974) 558.

[37] T. Balcerzak, K. Szałowski, M. Jaščur, A simple thermodynamic description of the combined Einstein and elastic models, J. Phys. Condens. Matter. 22 (2010) 425401.

[38] K. Singer, W. Smith, The classical and quantum-mechanical free energy of solid 
(lennard-jones) argon, Chem. Phys. Lett. 140 (1987) 406-410.

[39] C. Fan, D.D. Do, D. Nicholson, E. Ustinov, Chemical potential, Helmholtz free energy and entropy of argon with kinetic Monte Carlo simulation, Mol. Phys. 112 (2014) 6073.

[40] R.B. Stewart, R.T. Jacobsen, Thermodynamic Properties of Argon from the Triple Point to $1200 \mathrm{~K}$ with Pressures to $1000 \mathrm{MPa}$, J. Phys. Chem. Ref. Data. 18 (1989) 639-798.

[41] P.J. Linstrom, W.G. Mallard, NIST Chemistry webBook, NIST Standard Reference Database Number 69, 2014. 